\title{
Macroscopic properties of carbon nanotubes from molecular-mechanics simulations
}

\author{
A. Sears and R. C. Batra \\ Department of Engineering Science and Mechanics, MC 0219 Virginia Polytechnic Institute and State University, \\ Blacksburg, Virginia 24061, USA
}

(Received 18 August 2003; revised manuscript received 14 October 2003; published 11 June 2004)

\begin{abstract}
Results of molecular-mechanics simulations of axial and torsional deformations of a single wall carbon nanotube are used to find Young's modulus, the shear modulus, and the wall thickness of an equivalent continuum tube made of a linear elastic isotropic material. These values are used to compare the response of the continuum tube in bending and buckling with that obtained from the molecular mechanics simulations. It is found that the strain energy of bending deformation computed from the Euler-Bernoulli beam theory matches well with that obtained from the molecular-mechanics simulations. The molecular-mechanics predictions of the critical strains for axial buckling and shell wall buckling do not match well with those derived from the Euler buckling formula and the Donnell shell theory.
\end{abstract}

DOI: 10.1103/PhysRevB.69.235406

PACS number(s): 46.70.Lk

\section{INTRODUCTION}

Since their discovery in 1991 , both single wall and multiwall carbon nanotubes have become an active area of research. This is partly due to their having an extremely high specific strength and stiffness. These properties and their cylindrical shape allow for their potential applications in such diverse fields as fibrous reinforcement, atomic level piping, and nanostructures. The structural applications of carbon nanotubes require that we ascertain their macroscopic properties. Previous experimental and theoretical studies have tacitly presumed that they can be modeled as linear elastic and isotropic. Tables I and II summarize, respectively, values of Young's modulus $E$ computed by various investigators, from molecular mechanics (MM) simulations and experimental data. Except for the work of Yakobson et al., ${ }^{1}$ Halicioglu ${ }^{2}$ and Zhou et al., ${ }^{3}$ they all assume a wall thickness of $3.4 \AA$, which is the separation distance between adjacent walls in a multiwall nanotube (MWNT). The scatter in the experimental data is partly due to the poor resolution at these extremely small scales. There is less scatter in the values of $E$ computed from the MM simulations; $E$ so found is close to
$1 \mathrm{TPa}$. Yakobson et al. ${ }^{1}$ state that the $\pi$-bond length $0.66 \AA$ is a better choice for the wall thickness because it more properly models the wall buckling behavior of nanotubes.

When designing composites with carbon nanotubes as reinforcements, it is imperative that one replace a nanotube by an equivalent continuum structure such as a fiber or a hollow cylindrical tube. The response of the continuum structure to different deformations should closely mimic that of the carbon nanotube. Here we find a cylindrical tube made of a linear elastic isotropic material whose response to mechanical deformations is equivalent to that of a single wall carbon nanotube (SWNT); a schematic sketch is given in Fig. 1. The deformations of a SWNT are analyzed by MM simulations, and those of the cylindrical tube by commonly used engineering theories.

Two MM potentials, MM3 (Ref. 4) and the Tersoff-Brenner, ${ }^{5}$ are used to simulate deformations of a SWNT in tension, compression, torsion, bending, and buckling. Results from the tension and torsion tests are used to ascertain whether $E$ and the shear modulus $G$ vary with the strain. Values of $E$ and $G$ at zero strain in terms of the wall thickness $t$, changes in the diameter of a SWNT, and the

TABLE I. Values of Young's modulus computed from experimental data.

\begin{tabular}{lccccc}
\hline \hline \multicolumn{1}{c}{ Author(s) } & Year & $\begin{array}{c}\text { Modulus } \\
\text { (TPa) }\end{array}$ & $\begin{array}{c}\text { Deviation } \\
\text { (TPa) }\end{array}$ & Test method & Tube \\
\hline Treacy et al. (Ref. 6) & 1996 & 1.8 & 1.4 & Thermal vibrations & MWNT \\
Wong et al. (Ref. 7) & 1997 & 1.28 & 0.6 & Cantilever bending & MWNT \\
Krishnan et al. (Ref. 8) & 1998 & 1.3 & 0.5 & Thermal vibrations & SWNT \\
Salvetat et al. (Ref. 9) & 1999 & 0.81 & 0.41 & 3 point bending & BUNDLES \\
Salvetat et al. (Ref. 9) & 1999 & 1.28 & 0.59 & 3 point bending & MWNT \\
Tombler et al. (Ref. 10) & 2000 & 1.2 & na & 3 point bending & SWNT \\
Cooper and Young (Ref. 11) & 2000 & $0.78-2.34$ & na & Raman spectroscopy & SWNT \\
Yu et al. (Ref. 12) & 2000 & $0.27-0.95$ & na & Tension & MWNT \\
Lourie and Wagner (Ref. 13) & 1998 & $2.8-3.6$ & na & Raman spectroscopy & SWNT \\
Lourie and Wagner (Ref. 13) & 1998 & $1.7-2.4$ & na & Raman spectroscopy & MWNT \\
Yu et al. (Ref. 14) & 2000 & $0.32-1.47$ & na & Tension & Ropes \\
\hline \hline
\end{tabular}

${ }^{\mathrm{a}}$ Not available. 
TABLE II. Values of Young's modulus predicted from atomistic simulations.

\begin{tabular}{|c|c|c|c|c|c|c|}
\hline Author(s) & Year & $\begin{array}{l}\text { Modulus } \\
\text { (TPa) }\end{array}$ & $\begin{array}{c}\text { Thickness } \\
(\AA)\end{array}$ & $\begin{array}{c}\text { Poisson's } \\
\text { ratio } \nu\end{array}$ & Potential / Method & Trend \\
\hline Robertson et al. (Ref. 15) & 1992 & 1.06 & 3.4 & & Brenner \& $\mathrm{LDF}^{\mathrm{a}}$ & $1 / r^{2}$, helicity \\
\hline Yakobson et al. (Ref. 1) & 1995 & 5.5 & 0.66 & & Brenner & na \\
\hline Yakobson et al. (Ref. 1) & 1996 & 1.07 & 3.4 & 0.19 & Brenner & \\
\hline Cornwell and Wille (Ref. 16) & 1997 & 1 & 3.4 & & Brenner & $1 / r^{2}$ \\
\hline Halicioglu (Ref. 2) & 1997 & 0.5 & 6.8 & & Brenner & Radial \\
\hline Lu (MWNT) (Ref. 17) & 1997 & 1.11 & 3.4 & & Universal force field & Number of walls \\
\hline Lu (SWNT) (Ref. 17) & 1997 & 0.97 & 3.4 & & Universal Force field & None \\
\hline Hernandez et al. (Ref. 18) & 1998 & $1.24^{\mathrm{a}}$ & 3.4 & & Density-functional theory ${ }^{a}$ & None \\
\hline Yao and Lordi (Ref. 19) & 1998 & 1 & 3.4 & & Universal force field & $1 / r^{2}$ \\
\hline Ozaki et al. (Ref. 20) & 2000 & 0.98 & 3.4 & & Tight binding $\mathrm{O}(\mathrm{N})$ & None \\
\hline Van Lier et al. (Ref. 21) & 2000 & 1.09 & 3.4 & 0.11 & Hartree-Fock ${ }^{\mathrm{a}}$ & Helicity (small) \\
\hline Zhou et al. (Ref. 3) & 2000 & 5.1 & 0.71 & & Electronic band theory & $1 / r^{2}$ \\
\hline Belytschko et al. (Ref. 22) & 2002 & 0.94 & 3.4 & 0.29 & Modified Morse & \\
\hline
\end{tabular}

${ }^{\mathrm{a}}$ Quantum-mechanical method.

relation $E=2(1+\nu) G$, for an isotropic linear elastic material, are used to find $E, G, t$, and Poisson's ratio $\nu$. For simulations involving the bending and the buckling of a SWNT, these values of $E, G, \nu$, and $t$ are employed to compare the response predicted from the MM simulations with that given by the commonly used engineering approaches.

The paper is organized as follows. Section II describes briefly the potentials used for MM simulations. Techniques employed in virtual experiments used to analyze tensile, torsional, bending, and buckling deformations of a SWNT are described in Sec. III. Section IV gives results of MM simulations of tensile and torsional deformations and also lists expressions for Young's modulus and the shear modulus. The wall thickness and the elastic moduli of the cylindrical continuum tube are evaluated in Sec. V; these values are such that the mechanical response of the continuum tube in tensile and torsional deformations is equivalent to that of the SWNT. In Sec. VI, results of MM simulations for bending and buckling deformations of the SWNT are compared with that of the equivalent continuum tube derived from the Euler-Bernoulli beam theory and the Donnell shell theory. It is shown in Sec. VII that the strain energy of the combined tensile and torsional deformations of a SWNT equals the sum of the strain energies for individual deformations. For

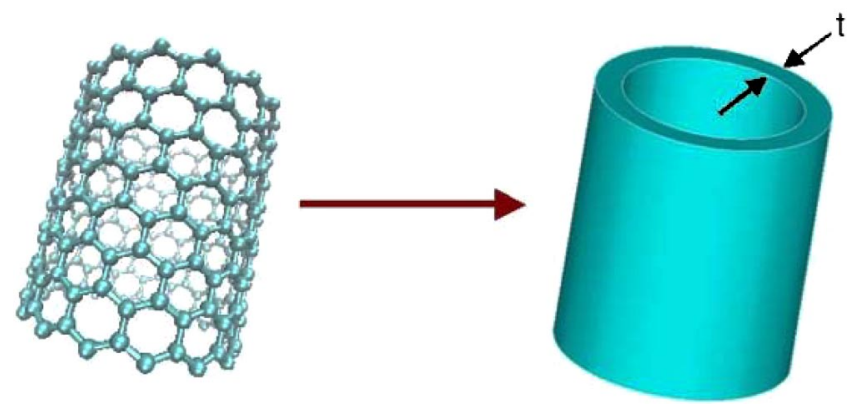

FIG. 1. (Color online) Single wall carbon nanotube and an equivalent cylindrical tube. the MM3 potential, contributions of different terms to the total strain energy are delineated in Sec. VIII. Characteristics of cylindrical continuum tubes equivalent in the mechanical response to SWNT's of different helicities are given in Sec. IX, and conclusions are summarized in Sec. X.

\section{POTENTIALS}

Two potentials used in this work are the MM3 (Ref. 3) and the Tersoff-Brenner. ${ }^{4}$ The MM3 potential is a class II pairwise potential with both higher-order polynomial expansions and cross terms; it is used primarily to model proteins. This potential is appropriate for carbon nanotubes due to the similarity of carbon bonding between the nanotube graphitic and the aromatic protein structures; the expression for the MM3 potential is given in the Appendix.

The Tersoff-Brenner potential is an empirical bond-order potential specifically designed for diamond and graphite structures. The bond strength is a pairwise potential function of the atomic separation, angle, and the number of bonds (neighbors). Rather than using a polynomial function to define the bond strength, the Tersoff-Brenner potential uses exponential functions similar to the Morse ${ }^{22}$ potential; the expression for the Tersoff-Brenner potential is given in the Appendix.

In our simulations, the carbon atoms in the MM3 potential were modeled $^{23}$ as alkene, or type 2 atoms, with the molecular-mechanics package Tinker. ${ }^{24}$ The potential energy of the structure is minimized through an adaptive minimization routine which utilizes either a truncated Newton or a negative curvature technique. Except where noted, all simulations used a nonbonded cutoff value of $30 \AA$, and minimized the potential energy to within $0.001 \mathrm{kcal} / \mathrm{mol} / \AA \mathrm{rms}$. The computer program BRENNERMD was used for simulations with the Tersoff-Brenner potential. These simulations minimized the Lagrangian using molecular-dynamics techniques with the temperature held at $300 \mathrm{~K}$.

The MM3, the Tersoff-Brenner, the Amber and the Morse 


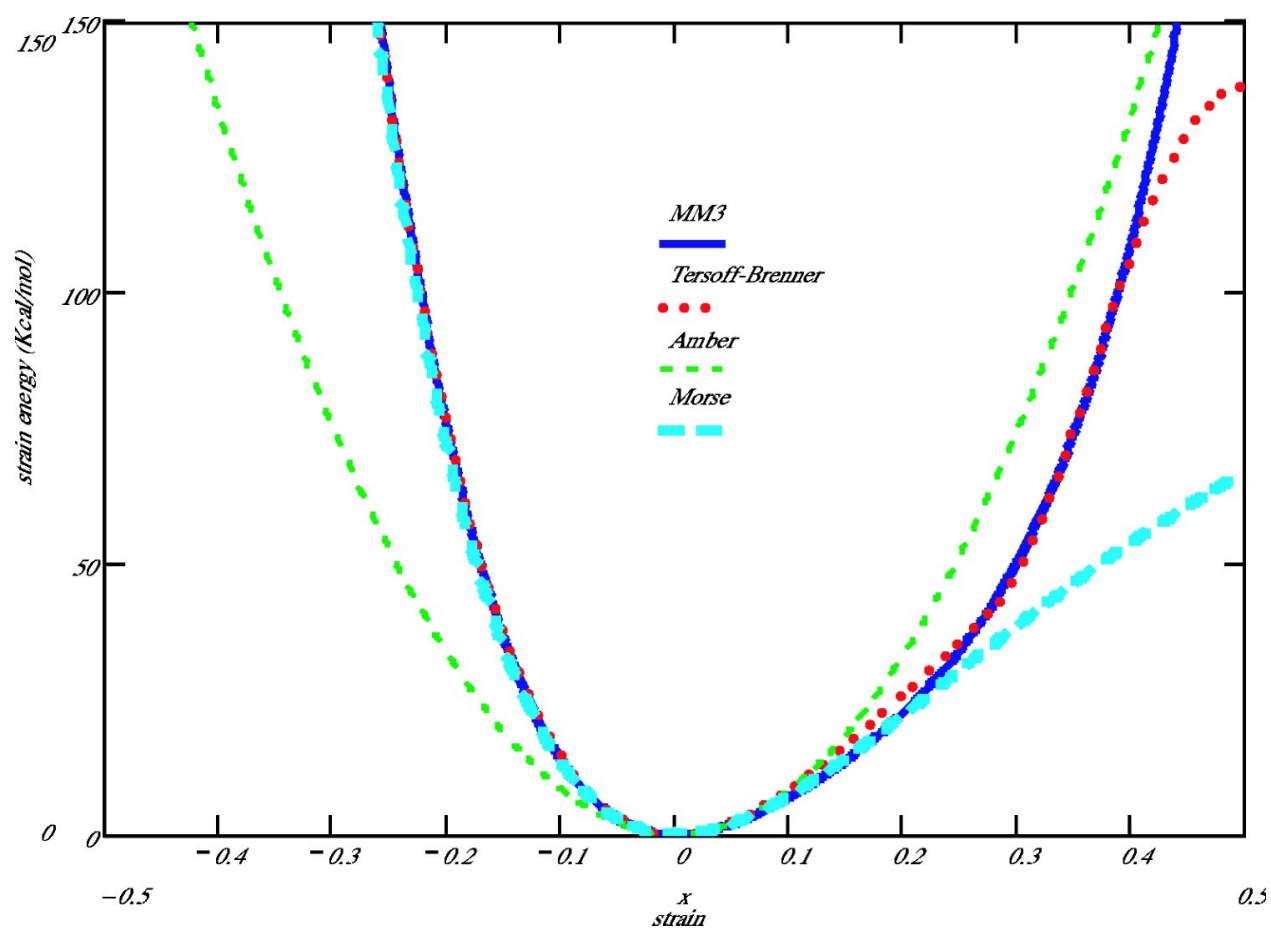

FIG. 2. (Color online) Variation with the bond strain of the Morse, the MM3, the TersoffBrenner, and the Amber bondstretching potentials.

bond-stretching terms for carbon-carbon $s p^{2}$ hybrid bonds are compared in Fig. 2 as a function of the bond strain. The bond strain equals the change in the bond length divided by the equilibrium bond length. Expressions for the Amber and the Morse potentials are only used in Fig. 2 so these expressions are not included; they can be found in Refs. 26 and 27. The Morse potential is generally believed to accurately model the covalent bonds. The bond-stretching terms of MM3 and Tersoff-Brenner potentials are very close to those of the Morse potential for bond strains up to $20 \%$. The Amber potential is a force-constant model, therefore is symmetric and parabolic about the vertical axis; it is accurate only for small strains. The MM3, the Tersoff-Brenner, and the Morse potentials have asymmetric variations with compressive deformations requiring more energy than tensile deformations of the same magnitude. Equations (A1) of the Appendix show that the MM3 bond-stretching potential is a quartic function of the bond strain; thus the moduli computed from it will depend upon the bond strain.

\section{VIRTUAL EXPERIMENTS}

The minimum-energy configuration for the molecular structure is first found. That is, all atoms are allowed to move freely until the total energy for the structure reaches a minimum. This minimum-energy configuration of the structure is henceforth referred to as the relaxed structure. For each increment in load, displacements for the deformation mode are estimated and applied to the relaxed structure. The appropriate boundary conditions for the specific deformation mode are applied by fixing positions of suitably selected atoms to maintain the prescribed displacement. Next the minimumenergy configuration for the loaded structure is found by allowing the remaining atoms to move until the total potential energy attains a minimum value. The strain energy due to deformation of the structure is determined by simply subtracting the energy of the relaxed structure from that of the loaded structure. From a plot of the strain energy vs a measure of deformation, effective parameters for a continuum model are derived.

Two SWNT models, involving infinite and finite lengths, were used. The infinite length tubes were modeled as short periodic tubes consisting of 256 atoms, whose bonds and interactions wrapped across the axial period. The nonbonded cutoff distance was reduced to $10 \AA$ so that atoms will not interact with each other both directly and across the periodic boundary. The periodic models were effective for quickly studying axial deformations, large compressive strains, and axial wall buckling. The finite length models were used to study the torsional, bending, and column buckling deformations. The ends of these nanotubes were left open which changes the bonding character of the structure and leads to edge effects. In order to mitigate these effects the boundary conditions were applied approximately one diameter length from the edge and the tube's aspect ratio (length/diameter) was kept above ten. Local effects, such as necking or swelling during axial deformation occur within $2 \AA$ of the atoms where boundary conditions were applied; these were found to have negligible effect on the total energy of the system.

Unless otherwise noted, all tests were performed on a $(16,0)$ (Ref. 28) SWNT. The diameters of the relaxed tubes were $11.87 \AA$ and $12.58 \AA$ for tubes modeled with the MM3 and the TB potentials, respectively.

\section{RESPONSE IN SIMPLE TESTS}

The axial deformation tests were performed on periodic SWNT's and did not require any boundary conditions. For 

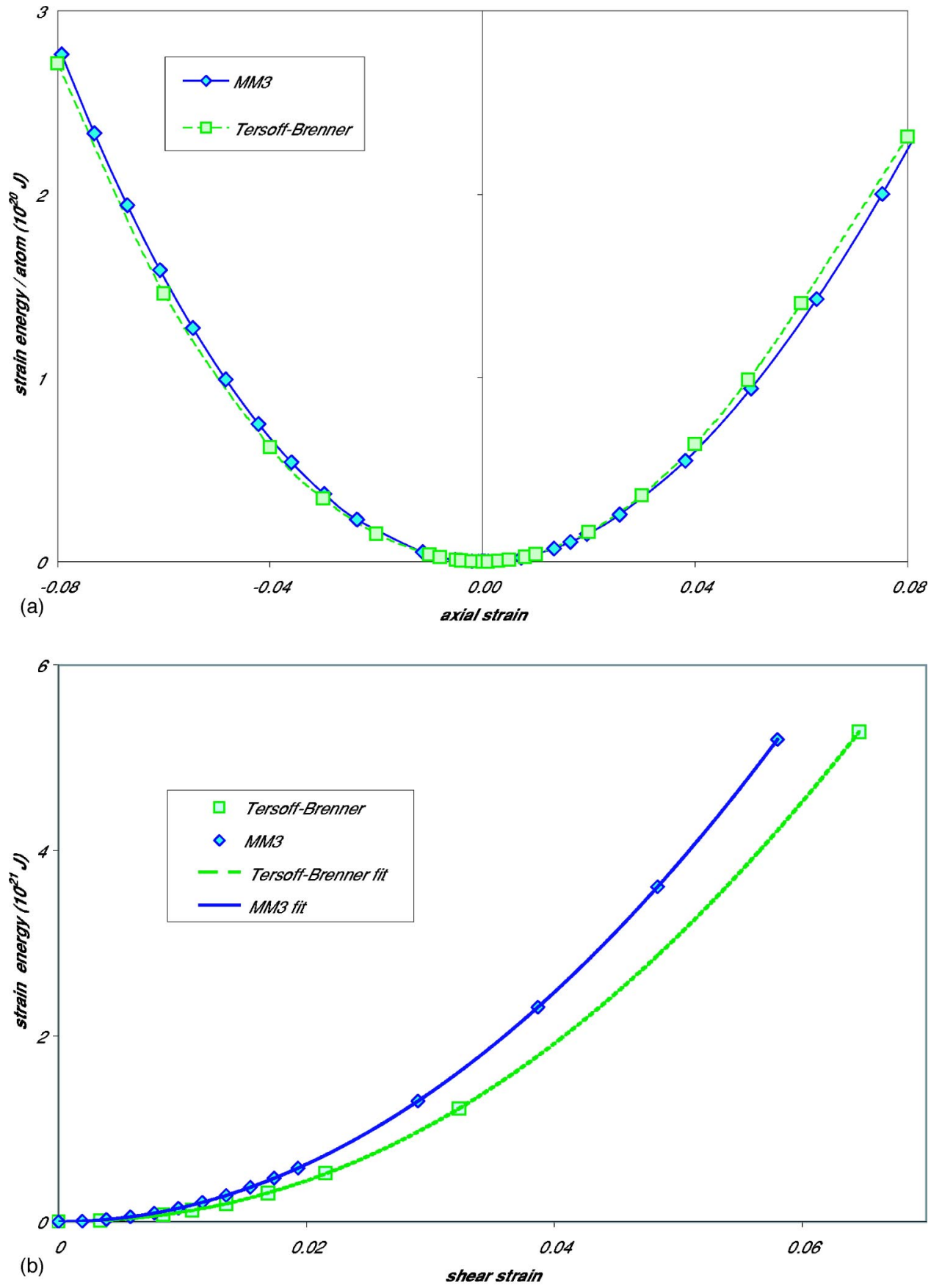

FIG. 3. (Color online) Strain energy results from (a) the axial, and (b) the torsional deformation of a SWNT with the MM3 and the Tersoff-Brenner potentials. the torsion test, boundary conditions were applied to two circumferential rings of atoms approximately one diameter away from the open ends. The minimum-energy configuration always coincided with zero axial deformation. Before the onset of buckling, circular cross sections remained circular for both the tension, compression, and torsion tests. The coefficients of variation (percent standard deviation) of the radial positions of atoms, for the relaxed and the $10 \%$ stretched configurations, were 0.05 and 0.12 , respectively. The diameter of the cross section of a tube deformed in torsion remained constant, but changed when it was deformed axially. Thus, Poisson's ratio was uniquely defined for each load step as the negative of the ratio of the lateral strain to the axial strain.
In a hollow cylindrical continuum tube equivalent to the SWNT, the simple tension and torsion tests would induce simple stress states of axial and shear stress, respectively. All of the strain energy is produced by a single stress component thereby allowing the axial and the shear moduli to be directly computed. The MM strain energy results for these tests, using both the MM3 and the Tersoff-Brenner potentials, are presented in Fig. 3. A smooth polynomial is fitted to the data points representing strain energies at different strains. The first derivative of this fit yields the corresponding stress component, and the second derivative gives the elastic modulus. If the degree of the best-fit polynomial is higher than two, then the elastic modulus will vary with the deformation. The lowest-order best-fit polynomial for the tension test data ob- 


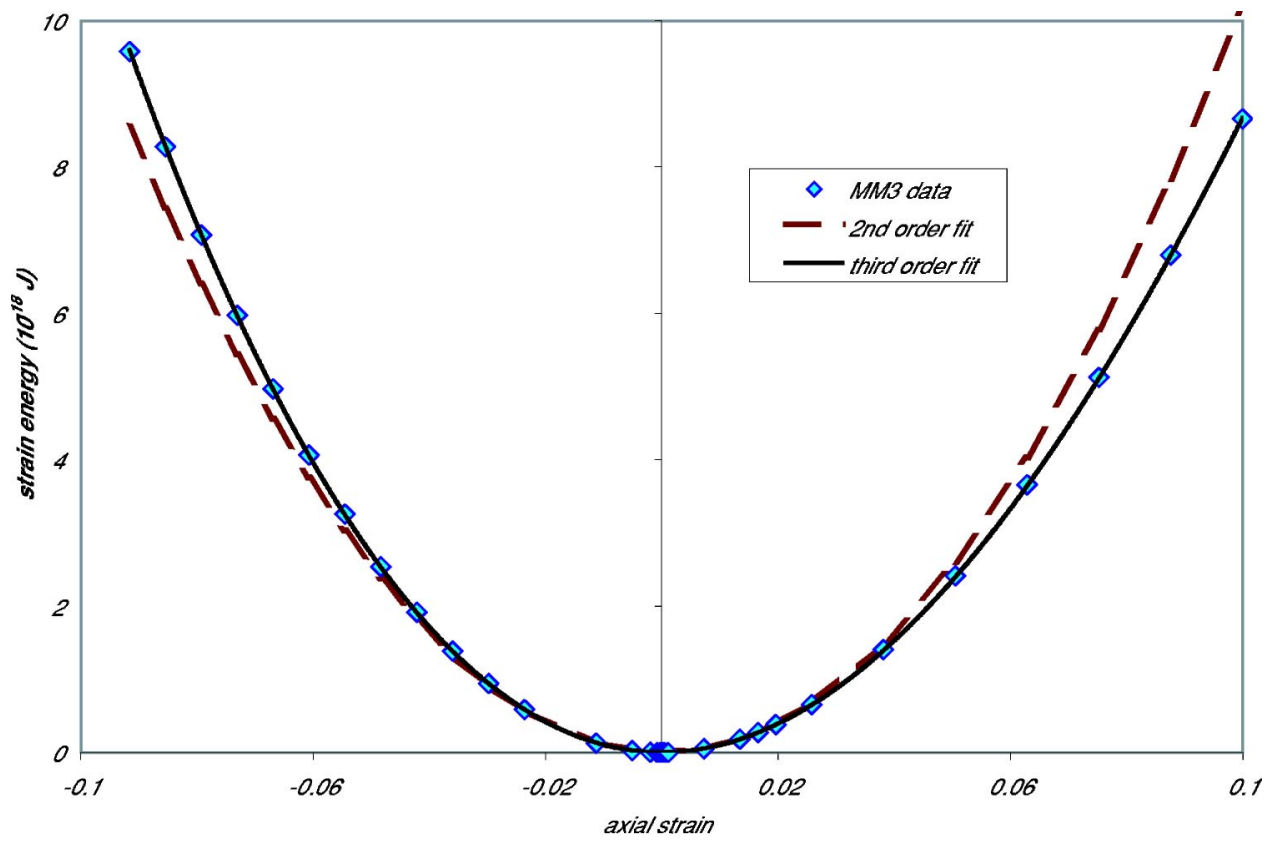

FIG. 4. (Color online) Comparison of the second- and the third-order fits to the MM3 strain energy results. tained with the MM3 potential is third order and is given by

$$
\begin{aligned}
W_{\nu}= & \left(-8.183 \times 10^{-7} \varepsilon^{3}+5.9 \times 10^{-7} \varepsilon^{2}\right. \\
& \left.-2.95 \times 10^{-12} \varepsilon\right) / A(t),
\end{aligned}
$$

where $W_{\nu}$ is the strain energy density in $J / \mathrm{m}^{3}, \varepsilon$ the nominal axial strain, and $A(t)$ the cross-sectional area, in $m^{2}$, is a function of the wall thickness $t$. Thus the axial modulus $E$ in Pascals is given by

$$
E=(-4.91 \varepsilon+1.18) 10^{-6} / A(t) .
$$

The third-order and the second-order polynomial fits have regression correlations of 1.0 and 0.996 , respectively. While both values are very high, the third-order fit more accurately describes the data, as shown in Fig. 4.

Young's modulus derived from the Tersoff-Brenner potential is given by

$$
E=(-5.33 \varepsilon+1.25) 10^{-6} / A(t) .
$$

The correlation coefficients of the second-order and the third-order polynomial fits are 0.997 and 1.0, respectively.

A procedure similar to the one described above for the tension test was followed for the torsion test. Values, in Pascal, of the shear moduli so found are

$$
\begin{gathered}
G=\left(86.4 \gamma^{2}+1.4536\right) 10^{-5} / J(t) \\
\text { for the Tersoff-Brenner potential, } \\
G=1.72 \times 10^{-5} / J(t) \quad \text { for the MM3 potential, }
\end{gathered}
$$

where $J$, the polar moment of inertia of the equivalent continuum tube, is a function of the wall thickness. Whereas deformations of the continuum tube are homogeneous for the simple tension test, they are inhomogeneous for the torsion test. The shear strain at points on the midsurface of the continuum tube was taken to equal that in the SWNT.
At zero strain, Young's moduli computed with the two potentials differ by about $6 \%$, while their variations with $\varepsilon$ are nearly the same. At $10 \%$ axial strain, Young's modulus derived from the MM3 potential has changed by over $58 \%$ of its value at zero strain. However, the shear moduli and Poisson's ratio do not necessarily share the same qualitative behaviors; Poisson's ratios computed from the results of the MM simulations are shown in Fig. 5. While the MM3 derived Poisson's ratio has a linear variation with the axial strain that derived from the Tersoff-Brenner potential varies nonlinearly for axial strains exceeding -0.02 .

We note that for a linear elastic isotropic material, $E, G$, and $\nu$ are constants and satisfy the relation $E=2 G(1+\nu)$.

\section{DETERMINATION OF THE WALL-THICKNESS OF THE EQUIVALENT CONTINUUM TUBE}

The problem of finding a continuum cylindrical tube whose response to axial and torsional deformations is identical to that of a $(16,0)$ SWNT is complicated by the fact that its Young's modulus and Poisson's ratio should vary with the axial strain but the shear modulus be either a constant or vary with the shear strain. In order to simplify the problem, it was decided that the equivalent continuum tube is made of a linear elastic isotropic material with mean diameter equal to the diameter of the SWNT, wall thickness $t$, and moduli equal to those of the SWNT at zero strain. Thus, in Eqs. (2)-(4),

$$
\begin{aligned}
& A(t)=\pi\left[\left(r_{c}+\frac{t}{2}\right)^{2}-\left(r_{c}-\frac{t}{2}\right)^{2}\right], \\
& \quad \text { and } J(t)=\frac{\pi}{2}\left[\left(r_{c}+\frac{t}{2}\right)^{4}-\left(r_{c}-\frac{t}{2}\right)^{4}\right],
\end{aligned}
$$

where $r_{c}$ is the radius of the SWNT. Substitution from Eqs. (2)-(4) into 


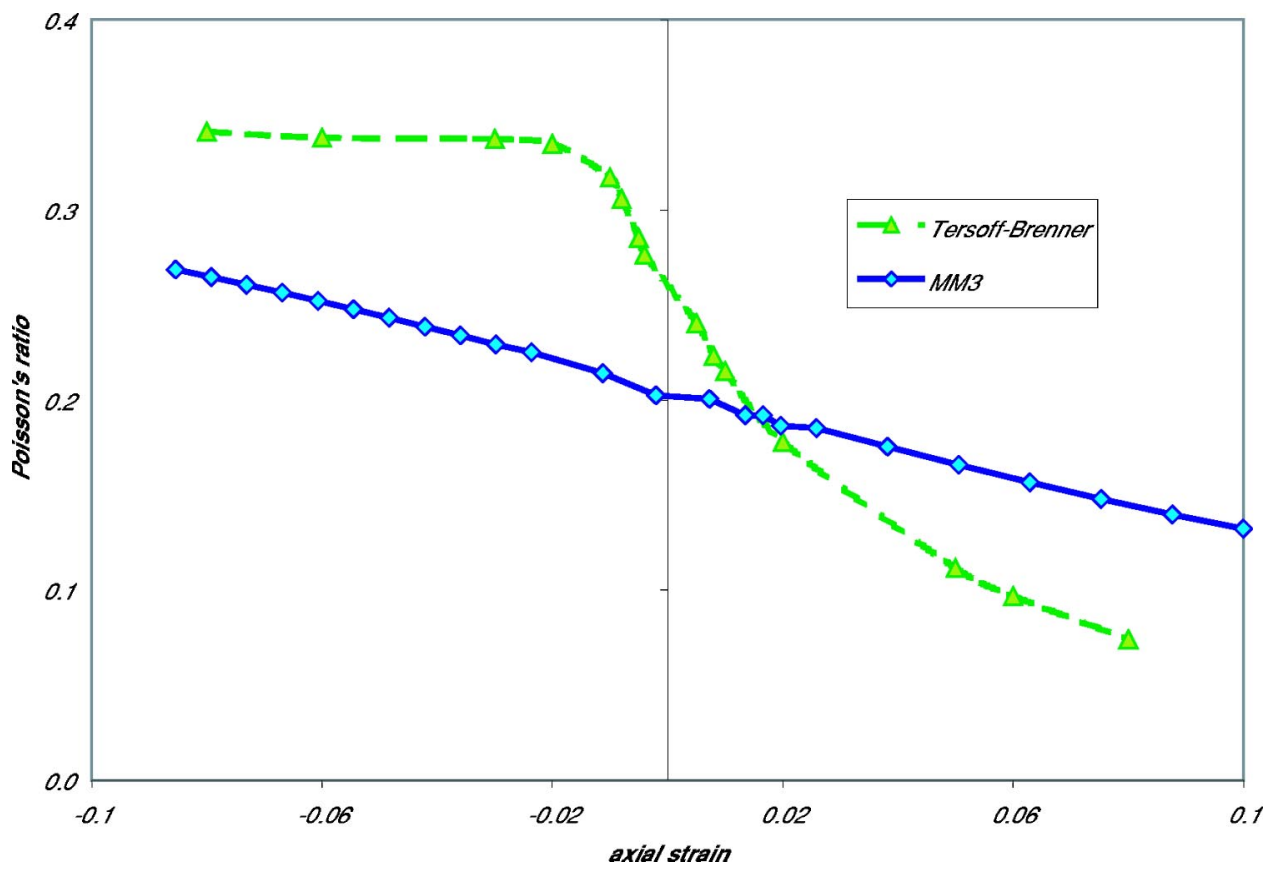

FIG. 5. (Color online) Variation with the axial strain of Poisson's ratio derived from the MM3 and the Tersoff-Brenner potentials.

$$
E(0, t)=2 G(0, t)[1+\nu(0)]
$$

gives an equation for the determination of the wall thickness $t$ of the equivalent continuum tube. Knowing $t, E$, and $G$ can be computed from Eqs. (2)-(4).

The results are presented in Table III for the MM3 and the Tersoff-Brenner potentials. The wall thickness thus found equals $1.34 \AA$ and $0.98 \AA$ for the MM3 and the TersoffBrenner potentials, respectively; it differs from the often used value of $3.4 \AA$, and the $0.66 \AA$ proposed by Yakobson et al.; ${ }^{1}$ had we used a wall thickness of $3.4 \AA$, then we would have obtained $E(0)$ to be $0.99 \mathrm{TPa}$ and $0.89 \mathrm{TPa}$, respectively, for the MM3 and the Tersoff-Brenner potentials which compare favorably with the values reported in the literature.

\section{BENDING AND BUCKLING OF A SWNT}

These simulations were done with the MM3 potential only.

\section{A. Bending of a Cantilever Beam}

The initial lateral displacements applied to the atoms of a $226 \AA$ long $(16,0)$ SWNT were estimated from the lateral deflection equation of the Euler-Bernoulli beam theory for a cantilever beam loaded by a point load at the unclamped edge. Axial displacements of atoms were estimated from the requirement that plane sections remain plane and perpen-

TABLE III. The wall thickness and elastic constants of an equivalent linear elastic continuum tube.

\begin{tabular}{lccccc}
\hline \hline \multicolumn{1}{c}{ Potential } & Structure & $\begin{array}{c}\text { Thickness } \\
(\AA)\end{array}$ & $E(\mathrm{TPa})$ & $G(\mathrm{TPa})$ & $\nu$ \\
\hline MM3 & $(16,0)$ & 1.34 & 2.52 & 0.96 & 0.21 \\
Tersoff-Brenner & $(16,0)$ & 0.98 & 3.10 & 0.81 & 0.26 \\
\hline \hline
\end{tabular}

dicular to the neutral axis. The boundary conditions used and the minimum-energy configuration found are shown in Fig. 6. The clamped end was modeled by fixing the locations of atoms on a circumferential ring approximately one diameter away from the open end. The point load was applied to three neighboring atoms on the top of the beam to avoid the tendency for wall indentation, which may occur when only a single atom is loaded. The axial positions of the loaded atoms were moved until the minimum-energy configuration was found.

The minimum-energy configurations were found to closely conform to those given by the Euler-Bernoulli beam theory. The strain energy for a linear elastic isotropic cantilever beam is given by

$$
W_{b}=\frac{3}{2} \frac{E I(t)}{L^{3}} \delta^{2}
$$

where $L$ is the length of the beam, $\delta$ represents the tip deflection, and $I$ denotes the area moment of inertia about the neutral axis;

$$
I=\frac{\pi}{4}\left[\left(r_{c}+\frac{t}{2}\right)^{4}-\left(r_{c}-\frac{t}{2}\right)^{4}\right] .
$$

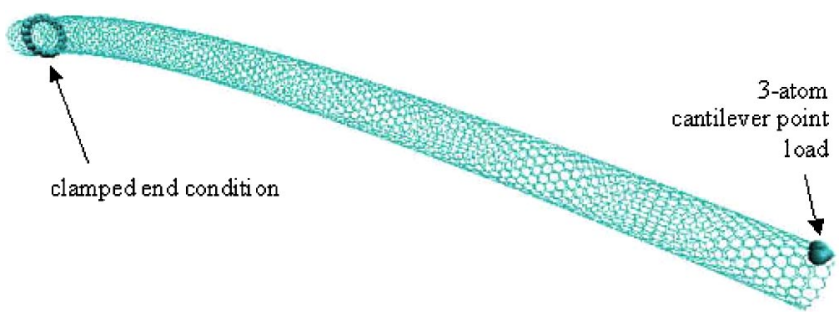

FIG. 6. (Color online) Deformed shape of a cantilever beam computed with the MM simulation employing the MM3 potential. 


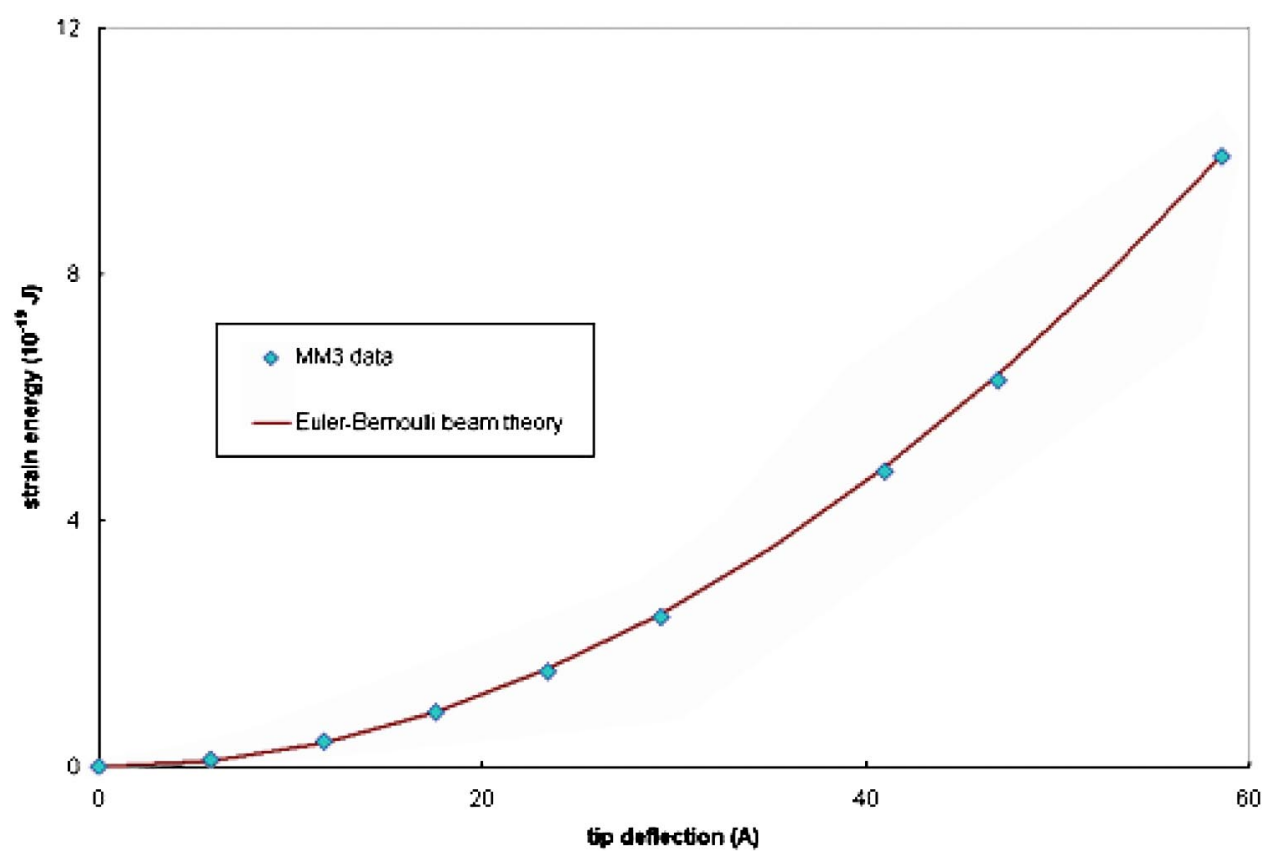

FIG. 7. (Color online) Comparison of the strain energy computed from the MM simulations with the MM3 potential with those obtained from the EulerBernoulli theory for the equivalent continuum tube.

Figure 7 compares the strain energy computed from the MM3 simulations with that given by Eq. (6). Thus the equivalent continuum tube mimics well the bending deformations of a SWNT.

\section{B. Buckling}

The column, axial shell wall, and torsional wall buckling were simulated by following the procedure employed for studying simple axial and torsional deformations. No initial perturbations were introduced to induce a buckling response. The four buckling modes found are shown in Fig. 8. A SWNT was assumed to have buckled when either the strain energy of deformations dropped significantly for an infinitesimal increase in the load or lateral deflections were very large. These invariably correspond to a noticeable increase in the number of iterations needed for the solution to converge.

\section{Column buckling}

A $(16,0)$ SWNT with an effective length of $171 \AA$ was used to study the column buckling. The boundary conditions used in the MM simulations closely resemble conditions for a clamped-clamped Euler column because the cross sections
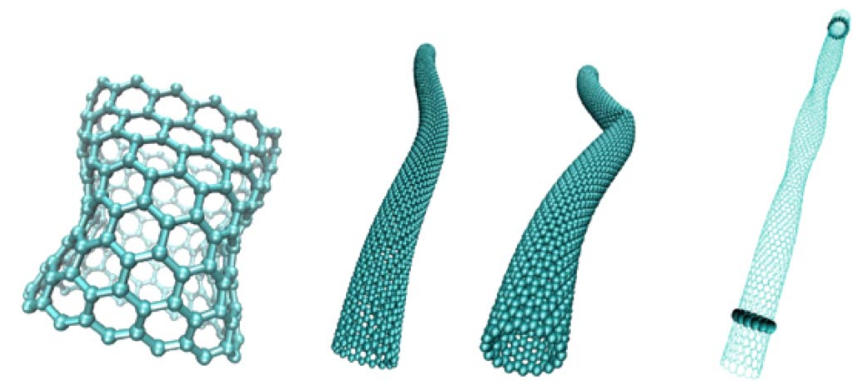

FIG. 8. (Color online) Four buckling modes found during the MM simulations: (a) shell wall, (b) columnar, (c) columnar with crimping, and (d) torsional (with boundary conditions highlighted). are not allowed to rotate. Comparing the critical buckling strain from the MM3 simulations with that for an Euler column provides an assessment of the equivalent continuum model. The critical strain

$$
\varepsilon_{\text {critical }}=4 \pi^{2} \frac{I(t)}{L^{2} A(t)}=\frac{\pi^{2}\left[\left(r_{c}+\frac{t}{2}\right)^{2}+\left(r_{c}-\frac{t}{2}\right)^{2}\right]}{L^{2}}
$$

for an Euler column is independent of the elastic modulus. For $L=171 \AA, \quad r_{c}=5.935 \AA$, and $t=1.34 \AA, \quad \varepsilon_{\text {critical }}$ $=0.024$ and is $16 \%$ less than that for the MM simulations. This could be due to not initially perturbing the SWNT.

\section{Shell wall buckling}

Two types of shell wall buckling, namely axial compression and torsional buckling, were studied. The Donnell shell theory was used to compute the buckling load for the cylindrical tube for both cases even though the ratio of the thickness to the mean radius $t / r_{c}=0.23$ is higher than the range of validity of the Donnell theory (e.g. see Yamaki ${ }^{25}$ ).

Axial Compression. The MM simulations of the axial wall buckling used periodic boundary conditions which most closely approximate simple supports. At an axial strain of $9.8 \%$, the SWNT buckled into two circumferential sinusoidal waves with a single axial wave as shown in Fig. 8(a).

According to the Donnell shell theory, the critical axial stress in a cylindrical tube is given by

$$
\sigma_{\mathrm{cr}}=\frac{1}{\sqrt{3(1-\nu)}} \frac{E t}{r_{c}} .
$$

Equation (8) is valid when the normal length $Z$ defined by

$$
Z=\left(1-\nu^{2}\right)^{1 / 2} \frac{L^{2}}{r_{c} t},
$$




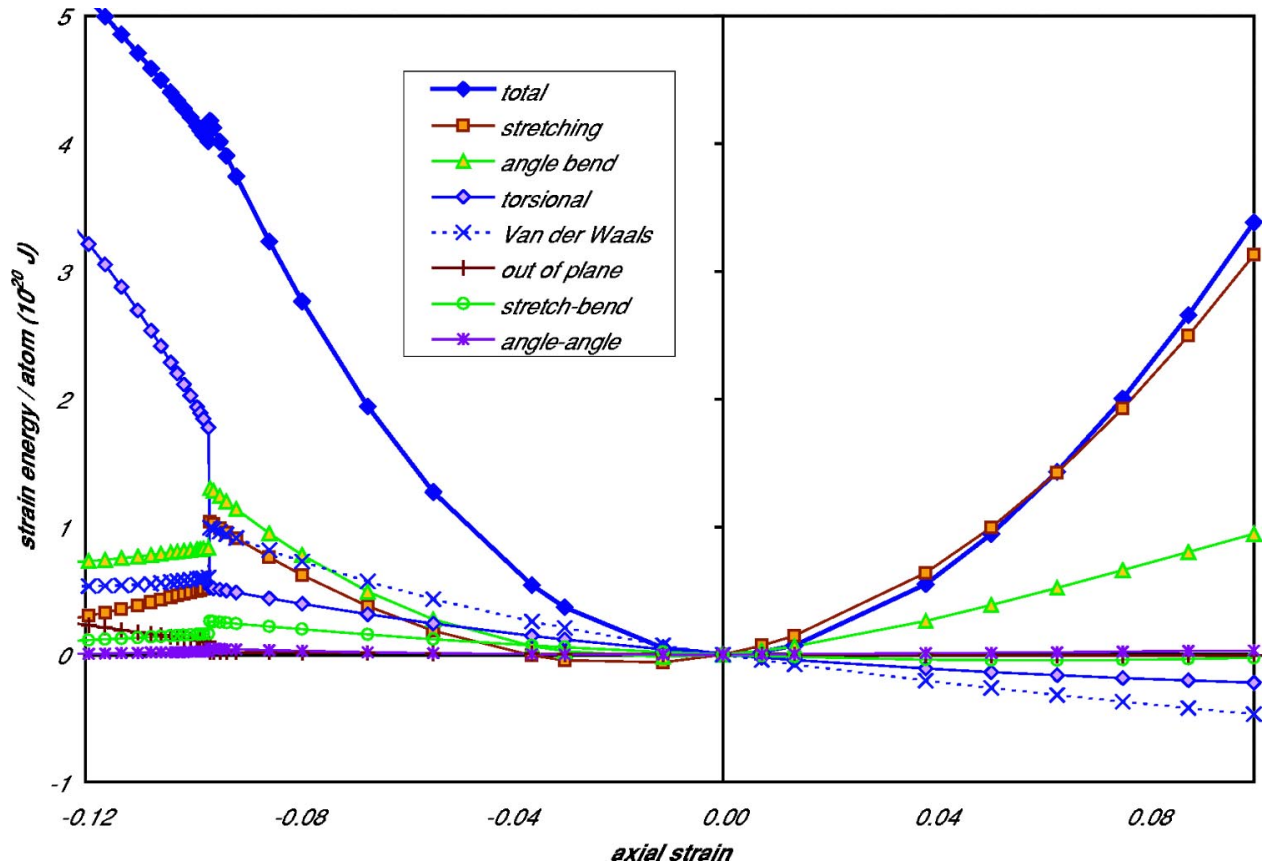

FIG. 9. (Color online) For the MM3 potential, variation with the axial strain of the energies of different modes of deformation. exceeds 2.85. The number $N$ of half-sine waves in the axial direction, and the number $m$ of sine waves in the circumferential direction are given by

$$
\begin{gathered}
N=\text { Integer }\left\{\left[\frac{3}{4}\left(1-\nu^{2}\right)\right]^{1 / 4} \sqrt{\frac{r_{c}}{t}}\right\}, \\
m=\text { Integer }\left\{\frac{(12)^{1 / 4}}{2 \pi} \sqrt{z}\right\},
\end{gathered}
$$

where integers closest to numbers given by the right-hand sides that yield the smallest buckling load should be used.

For the periodic model of the SWNT, $L=16.18 \AA, r_{c}$ $=5.937 \AA, t=1.34 \AA, \nu=0.21$; therefore, $Z=32, N=2$, $m=2$, and the axial strain at buckling $=0.147$. The MM simulations give $N=2$ and a critical strain of 0.098 . The shape of the midsection of the buckled SWNT resembled a peanut implying that $m=2$. Thus the axial strain from the Donnell shell theory does not match well with that from the MM simulations.

\section{Torsional buckling}

The MM simulations for the torsional deformations employed a $171 \AA$ long SWNT. The tube buckled at a shear strain of 0.064 and the buckled shape had two half-sine waves in the circumferential direction. For the equivalent continuum tube, $Z=3000$; thus it can be regarded as being infinitely long. According to the Donnell shell theory, the shear stress $\tau_{s}$ at buckling is given by

$$
\tau_{s}=\frac{\lambda_{s} D}{t / r_{c}^{2}}, \quad \lambda_{s}=2 \sqrt{2}(1-\nu)^{1 / 4}\left(\frac{r_{c}}{t}\right)^{1 / 2},
$$

where $D=E t^{3} / 12\left(1-\nu^{2}\right)$. Equations (11) give a shear strain of 0.066 which agrees well with that obtained from the MM simulations. However, for very long shells like the one being studied here, it is generally believed that the Donnell shell theory significantly underpredicts the buckling load and hence the shear strain at buckling.

One way to check the validity of the equivalent continuum tube for buckling deformations is to study the continuum problem with the finite element method. However, it has not been pursued here.

\section{COMBINED LOADING OF A SWNT}

Whether or not Young's and the shear moduli found in Sec. IV from MM simulations vary with the strain depends upon the degree of the best-fit polynomial. In order to check this and to further validate the equivalent continuum model, we simulated combined axial stretch and torsional deformations of a $(16,0)$ SWNT of effective length $171 \AA$, diameter $=11.87 \AA$ having 3072 atoms of which 2704 were strained. Several combinations of axial stretch and torsional deformations were simulated. The strain energies of the combined deformations were compared with that of the sum of individual deformations and also with the same deformations applied to the equivalent cylindrical tube. The maximum difference between the strain energy for the combined deformations and the sum of the strain energies of the individual deformations for the MM simulations was found to be less than $10 \%$ implying that the response of the tube up to $6 \%$ shear strain and $4 \%$ axial strain can be modeled as linear. Also, the difference in the strain energy of the continuum tube and that of the MM simulations was less than $10 \%$. Thus the equivalent continuum model described above is quite good for analyzing deformations of the SWNT.

\section{CONTRIBUTIONS TO THE STRAIN ENERGY FROM DIFFERENT TERMS IN THE MM3 POTENTIAL}

In order to better understand the differences in the response in axial tension and compression deformations of a 
TABLE IV. Parameters of cylindrical tubes equivalent in mechanical response to different SWNTs.

\begin{tabular}{lcccccccc}
\hline \hline \multicolumn{2}{c}{ SWNT } & & \multicolumn{5}{c}{ Equivalent cylindrical tube } \\
\cline { 1 - 5 } \cline { 5 - 8 } $\begin{array}{l}\text { Tube } \\
\text { structure }\end{array}$ & $\begin{array}{c}\text { Helicity } \\
(\text { deg })\end{array}$ & $\begin{array}{c}\text { Thickness } \\
(\AA)\end{array}$ & $\begin{array}{c}\text { Mean diameter } \\
(\AA)\end{array}$ & $\begin{array}{c}E \\
(\mathrm{TPa})\end{array}$ & $\begin{array}{c}G \\
(\mathrm{TPa})\end{array}$ & $\nu$ \\
\hline$(8,0)$ & 60 & & 1.34 & 5.97 & 2.31 & 1.03 & 0.19 \\
$(12,6)$ & 30 & & 1.35 & 11.79 & 2.43 & 0.99 & 0.21 \\
$(16,0)$ & 60 & & 1.34 & 11.88 & 2.52 & 0.96 & 0.21 \\
$(25,0)$ & 60 & & 1.34 & 18.55 & 2.49 & 0.97 & 0.21 \\
$(48,0)$ & 60 & 1.33 & 35.60 & 2.60 & 0.94 & 0.22 \\
$(10,10)$ & 0 & 1.38 & 12.85 & 2.41 & 1.01 & 0.22 \\
\hline \hline
\end{tabular}

SWNT with the MM3 potential, we have plotted in Fig. 9 the variation with the axial strain of different terms in the MM3 potential. For deformations involving an elongation of the tube, the energy due to bond stretching is almost equal to the total energy of the tube. The contribution to the total energy from the angle bend deformation is annulled by contributions from the van der Waals forces and torsional deformations. However, in axial compressive deformations, energies of deformations due to van der Waals forces, bond stretching, and angle bend modes of deformation are nearly the same. For the same value of the axial strain, the total strain energy of the tube is larger when it is deformed in compression than that when it is deformed in tension. Thus Young's modulus for compressive deformations is more than that for tensile deformations.

\section{EFFECT OF DIFFERENT WRAPPING INDICES}

MM simulations similar to those for a $(16,0)$ SWNT were performed on $(8,0),(10,10),(12,6),(25,0)$, and $(48,0)$ SWNT's and for each case, Young's modulus, the shear modulus, Poisson's ratio, and the wall thickness of the equivalent cylindrical tubes were determined. Results, summarized in Table IV, evince that the wrapping indices affect very little the values of the elastic moduli and the wall thickness of the equivalent cylindrical tube. For example, Young's modulus varies from 2.3 to $2.6 \mathrm{TPa}$, Poisson's ratio from 0.19 to 0.22 , and the wall thickness from 1.33 to $1.36 \AA$. Thus results for a $(16,0)$ SWNT are representative of those for tubes of other helicities and diameters.

\section{CONCLUSIONS}

We have used results of the molecular-mechanics simulations of a SWNT and the relation among Young's modulus, Poisson's ratio, and the shear modulus valid for a linear elastic isotropic material, to derive the thickness and values of the two elastic moduli of an isotropic linear elastic cylindrical tube equivalent to the SWNT. When the MM3 potential is used to simulate deformations of a SWNT, it is found that for the equivalent continuum tube, Young's modulus $=2.52$ $\mathrm{TPa}$, shear modulus $=0.96 \mathrm{TPa}$, Poisson's ratio $=0.21$, and wall thickness $=1.34 \AA$. The strain energy of bending deformations of the equivalent tube is found to match well with that computed from the molecular-mechanics simulations. Also strain energies of the combined axial and torsional deformations computed from the MM simulations matched well with that of the equivalent continuum tube. However, the responses of the SWNT and the cylindrical tube in buckling deformations where bending stiffness of the thin wall plays a noticeable role are found to be somewhat different. The helicity and the diameter of a SWNT have very little effect on the wall thickness, Young's modulus, and the shear modulus of the equivalent continuum tube.

\section{ACKNOWLEDGMENTS}

This work was financially supported by the AFOSR MURI grant to Georgia Institute of Technology, and by the ONR Grant No. N00014-98-1-0300, ARO Grant No. DAAD19-01-1-0657, and the NASA Space Consortium grant to Virginia Polytechnic Institute and State University.

\section{APPENDIX}

Variables $r, \theta$, and $\phi$ used below in Eqs. (A1) are shown in Fig. 10. A subscript 0 on a variable signifies its value in the unstressed equilibrium configuration. The total energy of a body equals the sum of the potential for all atoms in the body [the index $j$ in Eqs. (A1) ranges over bonded atoms, and the index $k$ over all atoms]. The MM3 potential is given by Eqs. (A1) in which terms $U_{s}, U_{\theta}$, and $U_{\phi}$ are the primary bond deformation terms; the term $U_{V d W}$ is the nonbonded van der Waals term; and $U_{s \phi}, U_{\phi s}$, and $U_{\theta \theta}$ represent cross interactions between the variables.

$$
\begin{gathered}
U=\sum_{i} \sum_{j}\left(U_{s}+U_{\theta}+U_{\phi}+U_{s \phi}+U_{\phi s}+U_{\theta \theta^{\prime}}\right) \\
+\sum_{i} \sum_{k} U_{V d W}, \\
U_{s}=71.94 K_{s}\left(r-r_{0}\right)^{2}\left[1-2.55\left(r-r_{0}\right)\right. \\
\left.+\left(\frac{7}{12}\right) 2.55\left(4-r_{0}\right)^{2}\right]
\end{gathered}
$$

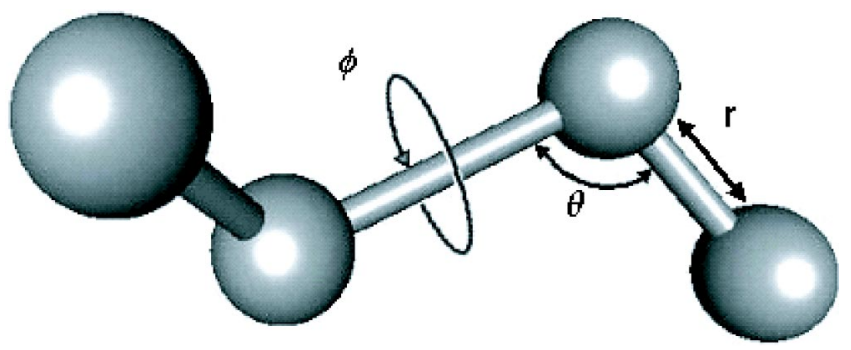

FIG. 10. Definitions of variables $r, \theta$, and $\phi$ used in defining pairwise potentials. 


$$
\begin{gathered}
U_{\phi}=\left(V_{1} / 2\right)(1+\cos \phi)+\left(V_{2} / 2\right)(1-\cos 2 \phi) \\
+\left(V_{3} / 2\right)(1+\cos 3 \phi), \\
U_{V d W}=\varepsilon_{0}\left\{-2.25\left(r_{\nu} / r\right)^{6}+1.84\left(10^{5}\right) \exp \left[-12.00\left(r / r_{\nu}\right)\right]\right\}, \\
U_{s \phi}=2.511 K_{s b}\left[\left(r-r_{0}\right)+\left(r^{\prime}-r_{0}^{\prime}\right)\right]\left(\theta-\theta_{0}\right), \\
U_{\phi s}=11.995\left(K_{\phi s} / 2\right)\left(r-r_{0}\right)(1+\cos 3 \phi), \\
U_{\theta \theta^{\prime}}=-0.021914 K_{\theta \theta^{\prime}}\left(\theta-\theta_{0}\right)\left(\theta^{\prime}-\theta_{0}^{\prime}\right) .
\end{gathered}
$$

Values of constants $K_{s}, K_{\theta}, V_{1}, V_{2}, V_{3}, \varepsilon_{0}, \gamma_{\nu}, K_{s b}$, $K_{\phi s}$, and $K_{\theta \theta}^{\prime}$ are given in Ref. 3 .

The Tersoff-Brenner potential reduces to the form given in Eqs. (A2) below for carbon-carbon bonds. The number of neighbors within a prescribed distance determines the number of bonds for an atom. The number of bonds, or bond order, helps define the bond strength of the pairwise bond potential.

$$
U=\sum_{i} \sum_{j(>i)} V_{R}\left(r_{i j}\right)-\bar{B}_{i j} V_{A}\left(r_{i j}\right)
$$

${ }^{1}$ B.I. Yakobson, C.J. Brabeck, and J. Bernholc, Phys. Rev. Lett. 76, 2511 (1995).

${ }^{2}$ T. Halicioglu, Thin Solid Films 312, 11 (1998).

${ }^{3}$ X. Zhou, J. Zhou, and Z. Ou-Yang, Phys. Rev. B 62, 13692 (2000).

${ }^{4}$ N.L. Allinger, Y.H. Yuh, and J.-H. Lii, J. Am. Chem. Soc. 111, 8551 (1989).

${ }^{5}$ D.W. Brenner, Phys. Rev. B 42, 9458 (1990).

${ }^{6}$ M.M.J. Treacy, T.W. Ebesen, and J.M. Gibson, Nature (London) 381, 678 (1996).

${ }^{7}$ E.W. Wong, P.E. Sheehan, and C.M. Lieber, Science 277, 1971 (1997).

${ }^{8}$ A. Krishnan, E. Dujardin, T.W. Ebbesen, P.N. Yianilos, and M.M.J. Treacy, Phys. Rev. B 58, 14013 (1998).

${ }^{9}$ J.-P. Salvetat, J.-M. Bonard, N.H. Thomson, A.J. Kulik, L. Torró, W. Benoit, and L. Zuppiroli, Appl. Phys. A: Mater. Sci. Process. 69, 255 (1999).

${ }^{10}$ T.W. Tombler, C. Zhou, L. Alexseyev, J. Kong, H. Dai, L. Liu, C.S. Jayanthi, M. Tang, and S-Y. Wu, Nature (London) 405, 769 (2000).

${ }^{11}$ C.A. Cooper, and R.J. Young, Proc. SPIE 4098, 172 (2000).

${ }^{12}$ M.-F. Yu, O. Lourie, M.J. Dyer, K. Moloni, and T.F. Kelly, Science 287, 637 (2000).

${ }^{13}$ O. Lourie and H. Wagner, J. Mater. Res. 13, 1471 (1998).

${ }^{14}$ M.F. Yu, B.S. Files, S. Arepalli, and R.S. Ruoff, Phys. Rev. Lett. 84, 5552 (2000).

$$
\begin{gathered}
V_{R}\left(r_{i j}\right)=f_{i j}\left(r_{i j}\right) D_{i j}^{c} /\left(S_{i j}-a\right) e^{-\sqrt{2 S_{i j}} \beta_{i j}\left(r_{i j} R_{i j}^{e}\right)}, \\
V_{A}\left(r_{i j}\right)=f_{i j}\left(r_{i j}\right) D_{i j}^{e} S_{i j} /\left(S_{i j}-1\right) e^{-\sqrt{2 S_{i j}} \beta_{i j}\left(r_{i j} R_{i j}^{e}\right)}, \\
B_{i j}=\left[1+\sum_{k(\neq i, j)} G_{i}\left(\theta_{i j k}\right) f_{i k}\left(r_{i k}\right) e^{\alpha_{i j k}\left[\left(r_{i j}-R_{i j}^{e}\right)-\left(r_{i k}-R_{i k}^{e}\right)\right]}\right]^{-\delta_{i}}, \\
G_{c}\left(\theta_{i j k}\right)=a_{0}\left\{1+c_{0}^{2} / d_{0}^{2}-c_{0}^{2} /\left[d_{0}^{2}+\left(1+\cos \theta_{i j k}\right)^{2}\right]\right\} .
\end{gathered}
$$

Here $r_{i j}$ is the distance between two atoms $i$ and $j$, and $\theta_{i j k}$ is the angle between lines joining atoms $i$ and $j$, and $i$ and $k$. The Tersoff-Brenner potential is not a function of the angle $\theta$ shown in Fig. 10. The functions $V_{R}\left(r_{i j}\right)$ and $V_{A}\left(r_{i j}\right)$ are the repulsive and the attractive potential energies, respectively, between atoms $i$ and $j$. The function $B_{i j}$ is the bond strength term, and $G(\theta)$ is the angle bond energy function. The functions $f_{i j}\left(r_{i j}\right)$ and $f_{i k}\left(r_{i k}\right)$ are the cutoff functions, which linearly reduce from 1 to 0 over a small range, to smooth out the cutoff of long-range atomic interactions. All other quantities are constants; their values can be found in Ref. 5 .

${ }^{15}$ D.H. Robertson, D.W. Brenner, and J.W. Mintmire, Phys. Rev. B 45, 12592 (1992).

${ }^{16}$ C.F. Cornwell and L.T. Wille, Solid State Commun. 101, 555 (1997).

${ }^{17}$ J.P. Lu, Phys. Rev. Lett. 79, 1297 (1997).

${ }^{18}$ E. Hernandez, C. Goze, P. Bernier, and A. Rubio, Phys. Rev. Lett. 80, 4502 (1998).

${ }^{19}$ N. Yao and V. Lordi, J. Appl. Phys. 84, 1939 (1998).

${ }^{20}$ T. Ozaki, Y. Iwasa, and T. Mitani, Phys. Rev. Lett. 84, 1712 (2000).

${ }^{21}$ G. VanLier, C. VanAlsenoy, V. Vandoren, and P. Geerlings, Chem. Phys. Lett. 326, 181 (2000).

${ }^{22}$ T. Belytschko, S.P. Xiao, G.C. Schatz, and R.S. Ruoft, Phys. Rev. B 65, 235430 (2002).

${ }^{23}$ A.R. Leach, Molecular Modeling, Principles and Applications, 2nd ed. (Prentice Hall, Harlow, 2001).

${ }^{24}$ J.W. Ponder, Computer Code TINKER MOLECUlar MODELING PACKAGE (2000).

${ }^{25}$ N. Yamaki, Elastic Stability of Circular Cylindrical Shells (NorthHolland, Amsterdam, 1984).

${ }^{26}$ P.A. Kollman (unpublished).

${ }^{27}$ R.E. Tuzun, D.W. Noid, B.G. Sumpter, and R.C. Merkle, Nanotechnology 7, 241 (1996).

${ }^{28}$ C.T. White, D.H. Robertson, and J.W. Mintmire, Phys. Rev. B 47, 5485 (1992). 